\title{
MiR-27a ameliorates inflammatory damage to the blood-spinal cord barrier after spinal cord ischemia: reperfusion injury in rats by downregulating TICAM-2 of the $\mathrm{TLR}_{4}$ signaling pathway
}

\author{
Xiao-Qian Li, Huang-Wei Lv, Zhi-Lin Wang, Wen-Fei Tan, Bo Fang and Hong Ma*
}

\begin{abstract}
Background: Spinal cord ischemia reperfusion (IR) injury causes inflammation and subsequently increases blood-spinal cord barrier leakage and Toll-like receptor $4\left(T L R_{4}\right)$ pathway activation. MicroRNAs (miRs) effectively regulate numerous target mRNAs during ischemia. However, their roles during IR injury are poorly understood. We investigated miRs involvement, particularly miR-27a, in $\mathrm{TLR}_{4}$ pathway-mediated inflammatory responses after IR.

Method: We used a genomics approach to examine changed miRs of rats that had undergone 14 minutes of ischemia, followed by 24 or 72 hours of reperfusion. Quantitative RT-PCR was used to identify and confirm the miRs involved in regulating $T L R_{4}$ pathway activation. We scanned miR databases for potential miR targets and confirmed these targets by quantitative RT-PCR. The miR mimic and anti-miR oligonucleotides (AMOs) were intrathecally injected at 12-hour intervals beginning three days before the ischemia. The effects of miRs on the $T_{L} R_{4}$ pathway and downstream cytokines were analyzed by PCR, western blotting, and ELISA. Double immunofluorescence staining was perfumed to determine the relationship between the targets and $T L R_{4}$. Blood-spinal cord barrier (BSCB) permeability was examined using Evans blue (EB) dye.
\end{abstract}

Results: A microarray analysis revealed that at 24 hours post-injury, three miRs were upregulated ( $>2.0$ fold) and 15 miRs were downregulated $(<0.5$ fold), and at 72 hours, four miRs were upregulated and 14 were downregulated compared to their levels in sham-operated controls. We focused on miR-27a, which is predicted to contain sequences complementary to the 3'-untranslated region (UTR) of Toll-like receptor adaptor molecule 2 (TICAM-2). Double immunostaining indicated that $\mathrm{TLR}_{4}$ activation correlated with changes in TICAM-2 expression. Compared to the rats in the IR and negative control groups, intrathecal infusion of the miR-27a mimic attenuated IR-induced TLR 4 activation and inflammatory damage to the BSCB, which was shown as decreased EB extravasation and lower levels of nuclear factor kappa-B (NF-KB) and Interleukin (IL)-1 $\beta$ at 24 and 72 hours after reperfusion, whereas pretreatment with miR-27a AMO aggravated these injuries.

Conclusions: We present the first evidence that miRs play an important role in spinal cord IR injury. We identified TICAM-2 as a novel target of miR-27a. miR-27a upregulation attenuates IR-induced inflammatory damage to the BSCB by negatively regulating TICAM-2 of the TLR ${ }_{4}$ signaling pathway and inhibiting the NF-KB/IL-1 $\beta$ pathway. These results provide new therapeutic targets for IR injury treatment.

Keywords: Blood-spinal cord barrier, MicroRNAs, MiR mimics, Anti-miR oligonucleotides, Toll-like receptor 4, Toll-like receptor adaptor molecule 2, Ischemia reperfusion injury

\footnotetext{
* Correspondence: mahong5466@yahoo.com

Department of Anesthesiology, First Affiliated Hospital, China Medical University, Address: No. 155 Nanjing North Street, 110001 Shenyang, Liaoning, China
}

\section{Biomed Central}

(C) $2015 \mathrm{Li}$ et al; licensee BioMed Central. This is an Open Access article distributed under the terms of the Creative Commons Attribution License (http://creativecommons.org/licenses/by/4.0), which permits unrestricted use, distribution, and reproduction in any medium, provided the original work is properly credited. The Creative Commons Public Domain Dedication waiver (http://creativecommons.org/publicdomain/zero/1.0/) applies to the data made available in this article unless otherwise stated. 


\section{Background}

Spinal cord ischemia reperfusion (IR) injuries have garnered much attention since 1986, when an IR injury was first reported, as they are associated with severe complications such as bladder, bowel, sexual dysfunction, and paraplegia [1]. Spinal cord IR injuries can induce a cascade of secondary events, such as neuronal or glial insults, that lead to further cell loss and behavioral impairments. These, in turn, are closely associated with inflammatory responses, including the release of cytokines, chemokines, and the recruitment of immune cells [2-4]. Numerous studies have shown that the major effectors involved in this inflammatory cascade activate the Toll-like receptors (TLRs), and the neuroprotective effect of inhibiting the $\mathrm{TLR}_{4}$ inflammatory pathway has been extensively studied by us and others [5-8].

We previously demonstrated that intrathecal antagonism of $\mathrm{TLR}_{4}$ in a rat model of IR, induced by transient occlusion of the aortic arch, could maintain the integrity of the blood-spinal cord barrier (BSCB) and attenuate secondary spinal cord injury by inhibiting proinflammatory cytokines, molecules downstream of the $\mathrm{TLR}_{4}$ pathway [6,7]. Contrary to previous investigations that showed that Myeloid differentiation factor $88\left(\mathrm{MyD}_{88)}\right.$ and MyD88 adaptor-like (Mal/TIRAP) adaptors functioned downstream of $\mathrm{TLR}_{4}$, the adapter TIR-containing adaptor molecule-2 (TICAM2, also known as TRAM) was shown to resemble $\mathrm{Mal} /$ TIRAP and to physically bridge it with TICAM-1 to functionally transmit the $\mathrm{TLR}_{4}$ signal $[9,10]$. Therefore, $\mathrm{TLR}_{4}$ recruits two crucial adaptors, TIRAP and TICAM-2, which are connected to two effective adapters, $\mathrm{MyD}_{88}$ and TICAM-1, respectively, after activation [9].

MicroRNAs (miRs) are small, non-coding RNAs that are capable of specific binding to one or more target mRNAs, and effectively regulate their post-transcriptional expression in various tissues [11-13]. Studies have shown that several miRs can dramatically alter normal physiological processes and are involved in the pathogenesis of various diseases $[14,15]$. Recent studies showed that ischemia alters the expression of miRs in cardiac tissue, and antagomirs against miRs improved neovascularization and augmented functional recovery in a large animal model of cardiac IR injury, suggesting a role for miRs in the regulation of IR [16,17]. Furthermore, there is also increasing evidence for the involvement of miRs in traumatic spinal cord injury $[18,19]$. However, the role of miRs in IR is not well understood. Exploring the expression profiles of miRs altered after IR might reveal whether miR-dependent post-transcriptional gene regulation in $\mathrm{TLR}_{4}$-mediated inflammation determines the progression of, and recovery from, secondary damage to the spinal cord.

In this study, we first used miR arrays to determine the expression patterns of miRs in a rat model of spinal cord IR injury, and then identified their target mRNAs by searching the TargetScan, MicroCosm Targets (version 5), and microRNA.org databases. Among the miRs identified, we observed that $m i R-27 a$ was one of the most dysregulated miRs, and further defined TICAM-2, a key regulator of the $\mathrm{TLR}_{4}$ pathway, as its target. Then, the effects of $m i R-27 a$ were assessed in a rat model of IR by intrathecal pretreatment with an $\mathrm{miR}$ mimic and an anti-miR oligonucleotide (AMO) starting three days before ischemia. Our results demonstrated that increasing the expression of $m i R-27 a$ attenuated IR-induced spinal cord injury by negatively modulating the $\mathrm{TLR}_{4}$ signaling regulator TICAM-2, which may be a new therapeutic target under neuroinflammatory conditions.

\section{Materials and methods}

\section{Experimental animals}

The experimental procedures were performed in accordance with the Guide for the Care and Use of Laboratory Animals (United States National Institutes of Health publication number 85-23, National Academy Press, Washington DC, revised 1996) The animals used in this study were male Sprague-Dawley rats (obtained from Animal center of China Medical University, Shenyang, China) weighing between 200 and $250 \mathrm{~g}$. The rats were bred in standard cages with free access to food and water, and were housed separately after surgery at First Affiliated Hospital of China Medical University.

\section{Rat model of spinal cord ischemia reperfusion injury}

To establish the spinal cord IR model, IR was induced by occluding the aortic arch for 14 minutes, as previously reported [6,7]. In brief, rats were anesthetized with an intraperitoneal injection of $4 \%$ sodium pentobarbital (Beyotime Biotechnology, Shanghai, China) at a dose of $50 \mathrm{mg} / \mathrm{kg}$, and then the aortic arch was exposed through a cervicothoracic approach. Under direct visualization, the aortic arch was cross-clamped between the left common carotid artery and left subclavian artery. Occlusion was confirmed with a laser Doppler blood flow monitor (Moor Instruments, Axminster, Devon, United Kingdom). Ischemia, which is defined as a 90\% decrease in the flow measured at the femoral artery\{AU Query: If this definition originates from any official published guidelines, please provide a reference\}, continued for 14 minutes, after which the clamps were removed and reperfusion was allowed to continue for either 24 or 72 hours. Sham-operated rats underwent the same procedure without aortic arch occlusion.

\section{MiR microarray analysis}

To assess $\mathrm{MiR}$ expression in the spine, $\mathrm{L}_{4-6}$ segments of the spinal cord were harvested at 24 and 72 hours after reperfusion, frozen in liquid nitrogen, and stored at $-80^{\circ} \mathrm{C}$ until use. Total RNA was isolated from the samples using TRIzol $^{\circledR}$ reagent (Invitrogen, Carlsbad, California, United 
States) and the miRNeasy mini kit (Qiagen, West Sussex, United Kingdom) according to the manufacturers' instructions. After measuring the quantity of RNA using a NanoDrop 1000 (Youpu Scientific Instrument Co., Ltd., Shanghai, China), the samples were labeled using the miRCURY ${ }^{\text {m }}$ $\mathrm{Hy}{ }^{\mathrm{rm}} / \mathrm{Hy}^{5 \mathrm{sm}}$ Power labeling kit (Exiqon, Vedbaek, Denmark) and hybridized on a miRCURY ${ }^{\mathrm{m}}$ LNA Array (version 18.0, Exiqon, Vedbaek, Denmark). After washing, the slides were scanned using an Axon GenePix 4000B microarray scanner (Axon Instruments, Foster City, California, United States). Scanned images were then imported into the GenePix Pro 6.0 program (Axon Instruments) for grid alignment and data extraction. Replicated miRs were averaged, and miRs with intensities of 50 or more in all samples were used to calculate a normalization factor. Expressed data were normalized by median normalization. After normalization, the miRs that were significantly differentially expressed were identified by Volcano Plot filtering. Finally, hierarchical clustering was performed to determine the differences in the miR expression profiles among the samples by using MEV software (version 4.6; TIGR, Microarray Software Suite 4, Boston, United States).

\section{Measurement of Evans blue extravasation}

After 24 and 72 hours of reperfusion, Evans blue dye ((EB) 30 g/L; Sigma-Aldrich, Louis, United States) was

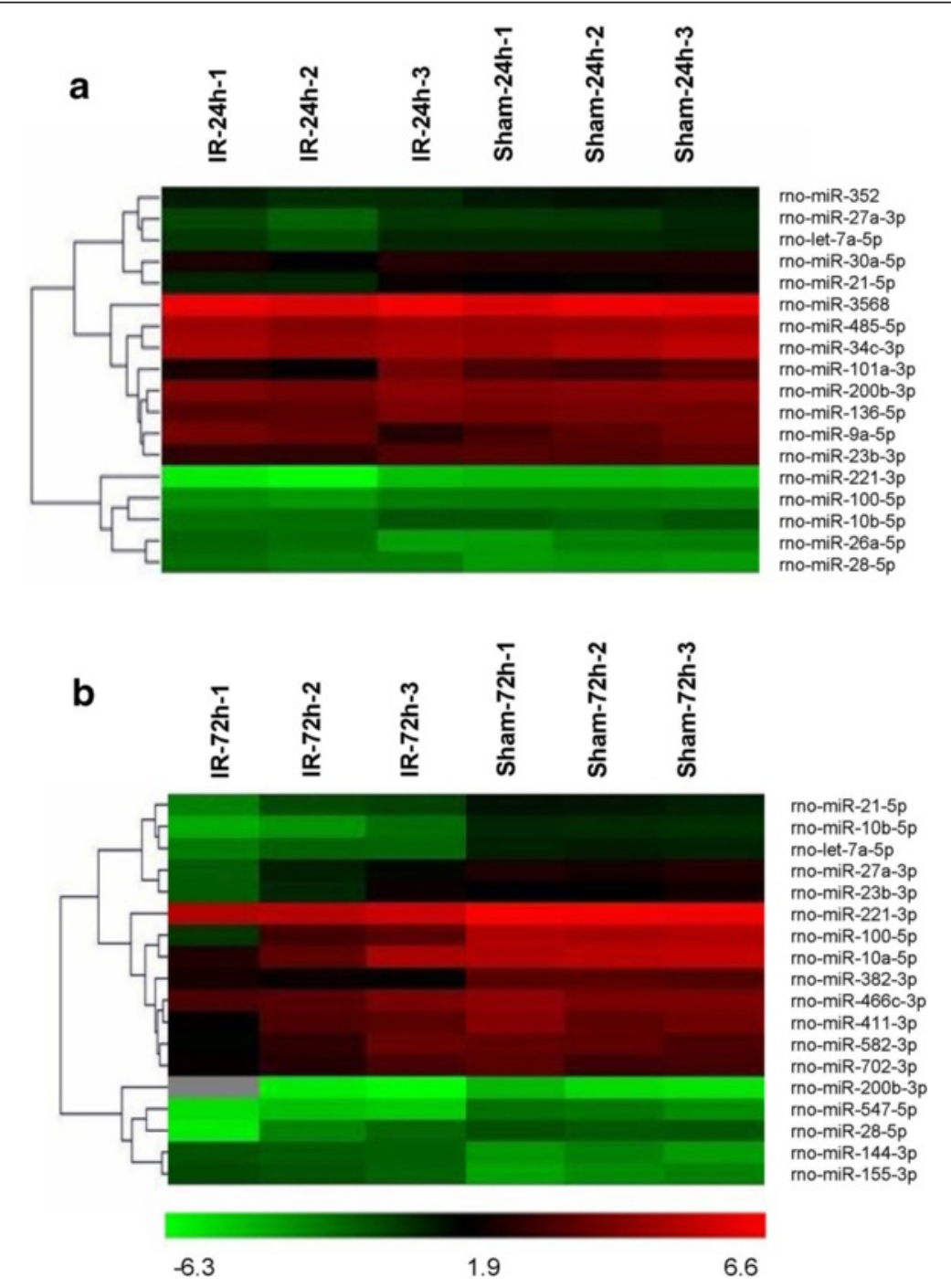

Figure 1 Hierarchical cluster analyses of altered microRNAs (miRs) after spinal cord ischemia reperfusion (IR) injury. (a) Differentially expressed miRs in the sham-operated and IR groups at 24 hours post-injury ( $n=$ three per group). (b) Differentially expressed miRs in the sham-operated and IR groups at 72 hours post-injury ( $n=$ three per group).Each row represents an miR and each column represents a sample. The color code shown at the bottom of the heat maps is linear, with green as the lowest and red as the highest. The miRs that were upregulated are shown in green to red, whereas the miRs that were downregulated are shown from red to green. Among all the significantly changed miRs, miR-27a was the most significantly downregulated in injured spinal cords at the abovementioned time points. 
intravenously injected $(45 \mathrm{mg} / \mathrm{kg})$ into the tail vein 60 minutes before the animals were euthanized. After adequate perfusion with saline under deep anesthesia, the $\mathrm{L}_{4-6}$ segments were removed, soaked in methanamide (Beyotime Biotechnology, Shanghai, China) for 24 hours at $60^{\circ} \mathrm{C}$, and then centrifuged. $\mathrm{EB}$ content was measured as the absorbance of the supernatant at $632 \mathrm{~nm}$ on a microplate reader (BioTek, Winooski, Vermont, United States) and is reported as the amount of EB per wet tissue weight $(\mu \mathrm{g} / \mathrm{g})$. To measure the fluorescence, the tissue was fixed in $4 \%$ paraformaldehyde (Beyotime Biotechnology, Shanghai, China), sectioned $(10 \mu \mathrm{m})$, sealed in a light-tight container, and frozen. EB staining was visualized using a BX-60 fluorescence microscope (Olympus, Melville, New York, United States) with a green filter.

\section{Quantification of miR expression}

MiR expression was quantified by using an Applied Biosystems 7500 Real-Time PCR System (Foster City, California, United States) to verify regulation of the miR targets in the spinal segments of the IR and sham groups. Total RNA from the $\mathrm{L}_{4-6}$ segments of the spinal cords was extracted with TRIzol reagent and reverse transcribed to cDNA with the PrimeScript ${ }^{\circ}$ miRNA cDNA synthesis kit (Perfect Real Time; TaKaRa, Dalian, China) according to the manufacturers' instructions. PCR was then used to amplify miR-27a using SYBR Premix Ex TaqTM II (Perfect Real Time; TaKaRa, Tokyo, Japan) and miR-27a-specific primers (forward, 5'-ACACTCCAGCTGGGTTCACAGTGGCTAAG-3' and reverse, 5'-TGGTGTCGTG GAGTCG-3'; RiboBio, Guangzhou, China) at $95^{\circ} \mathrm{C}$ for 10 seconds, followed by 40 cycles of $95^{\circ} \mathrm{C}$ for five seconds and $60^{\circ} \mathrm{C}$ for 20 seconds. The primers used to amplify U6 were $5^{\prime}$-CTCGCTTCGGCAGCACA-3' (forward) and 5'-AACGCTTCACGAATTTGCGT-3' (reverse). All reactions were performed in triplicate. The relative expression of $m i R-27 a$ was normalized to U6. Data were analyzed by using the $2^{-\Delta \Delta \mathrm{Ct}}$ method.

\section{Intrathecal pretreatment with a synthetic miR mimic and an anti-miR oligonucleotide}

The method used to pretreat rats with a mimic and an AMO of miRNA-27a (GenBank number: [NR_031833.1]) and negative controls has been previously described [17]. For intrathecal infusion, a laminectomy was performed at the level of the thoracic vertebrae under pentobarbital anesthesia (Beyotime Biotechnology, Shanghai, China).

Table 1 MicroRNAs (miRs) that were differentially expressed in the spinal cord of rats 24 hours after ischemia reperfusion (IR) injury compared with sham-operated rats ( $n=$ three per group)

\begin{tabular}{|c|c|c|c|c|c|c|}
\hline \multirow[b]{2}{*}{$\mathrm{miR}$} & \multicolumn{2}{|c|}{ Average intensity of all probes (normalized) } & \multicolumn{2}{|c|}{ Standard deviation } & \multirow[b]{2}{*}{ Fold change } & \multirow[b]{2}{*}{$P$ value } \\
\hline & IR-24 hours & Sham-24 hours & IR-24 hours & Sham-24 hours & & \\
\hline \multicolumn{7}{|l|}{ Upregulated } \\
\hline rno-miR-3568 & 1.1817 & 0.3304 & 0.7156 & 0.3879 & 3.5766 & 0.0460 \\
\hline rno-miR-34c-3p & 0.1437 & 0.6822 & 0.4047 & 0.8048 & 4.7460 & 0.1664 \\
\hline rno-miR-200b-3p & 0.0201 & 0.1628 & 0.1434 & 0.7703 & 8.1081 & 0.1200 \\
\hline \multicolumn{7}{|l|}{ Downregulated } \\
\hline rno-miR-352 & 2.0747 & 0.6335 & 0.2746 & 1.2941 & 0.3053 & 0.0667 \\
\hline rno-miR-27a-3p $p^{a}$ & 1.1155 & 0.4575 & 0.0362 & 0.7307 & 0.4101 & 0.0276 \\
\hline rno-let-7a-5p $p^{a}$ & 1.1155 & 0.5024 & 0.0362 & 1.1760 & 0.4504 & 0.1449 \\
\hline rno-miR-30a-5p & 5.8525 & 1.8522 & 0.0380 & 1.2318 & 0.3164 & 0.0390 \\
\hline rno-miR-21-5p & 3.7968 & 1.2200 & 0.1440 & 1.1157 & 0.3213 & 0.0383 \\
\hline rno-miR-485-5p & 0.5746 & 0.3074 & 0.1272 & 0.2713 & 0.4954 & 0.0140 \\
\hline rno-miR-136-5p & 26.647 & 10.0652 & 0.0403 & 1.0331 & 0.3777 & 0.0515 \\
\hline rno-miR-9a-5p & 30.965 & 12.0872 & 0.1480 & 0.8460 & 0.3901 & 0.0433 \\
\hline rno-miR-101a-3p & 20.289 & 10.5901 & 0.0886 & 0.4960 & 0.4919 & 0.0389 \\
\hline rno-miR-10b-5p $p^{a}$ & 17.175 & 7.4396 & 0.0651 & 0.8409 & 0.4331 & 0.0567 \\
\hline rno-miR-23b-3pa & 10.737 & 5.2797 & 0.0318 & 0.8743 & 0.4918 & 0.1107 \\
\hline rno-miR-221-3p & 0.5148 & 0.1355 & 0.1642 & 1.0404 & 0.3634 & 0.0162 \\
\hline rno-miR-26a-5p & 1.9140 & 1.1414 & 0.1408 & 0.5557 & 0.4959 & 0.1241 \\
\hline rno-miR-100-5p & 2.6130 & 1.1216 & 0.1617 & 0.6810 & 0.4293 & 0.0415 \\
\hline rno-miR-28-5p & 0.0722 & 0.0286 & 0.2262 & 1.3509 & 0.3969 & 0.1642 \\
\hline
\end{tabular}

Only the miRs with fold changes that were significantly different (upregulated $>2.0$ fold or downregulated $<0.5$ fold; $P<0.05$ ) are shown. \#\#: ${ }^{\mathrm{a}} \mathrm{miRs}$ that were differentially expressed in the spinal cord at both 24 and 72 hours after IR. 
A polyethylene catheter (PE10, Portex, Kent, United Kingdom, inside diameter (ID): $0.28 \mathrm{~mm}$ and outside diameter (OD): $0.61 \mathrm{~mm}$ ) was passed caudally from $\mathrm{T}_{9-12}$, and $2 \mathrm{~cm}$ of the free end was left exposed in the upper thoracic region. We intrathecally infused $100 \mu \mathrm{L}$ of a synthetic miR-27a mimic (mimic-27a), an AMO (AMO-27a), or the negative control $(N C-27 a$, all at $50 \mathrm{mg} / \mathrm{kg}$; Jima Inc., Shanghai, China) pretreated with Lipofectamine 2000 (Invitrogen) continuously for three days before the surgical operation. The sequences of mimic-27a, $A M O-27 a$, and $N C-27 a$ were $5^{\prime}$-UUCACAGUGGCUA AGUUCCGC-3', 5'-GCGGAACTTAGCCACTGTGAA3', and 5'-AAGGCAAGCUGACCCUGAAGUU-3', respectively. To analyze the specificity and efficacy of the miR-27a and AMO-27a, real-time PCR was performed as described above.

\section{Quantification of $\mathrm{TLR}_{4}$ and TICAM-2 mRNA}

Quantitative real-time PCR was used to detect $\mathrm{TLR}_{4}$ and TICAM-2 mRNA as previously described [7]. Total RNA was extracted from $\mathrm{L}_{4-6}$ spinal cord tissue using TRIzol reagent according to the manufacturer's instructions. PCR was performed as described previously using SYBR Green SuperMix-UDG on a Prism 7000 Sequence Detection
System (Applied Biosystems) and the following primers: TLR $_{4}$ (NM_0191178) forward, 5'-GGATGATGCCTCTCT TGCAT- ${ }^{\prime}$ ' and reverse, $5^{\prime}$-TGATCCATGCATTGGTAGG TAA-3'; TICAM-2 (NM_021649) forward, 5'-GGGAATTCATAATGGGTATCGGGAAGTC-3' and reverse, 5'-GG CTGCAGGTTATATGTTTCATCTCAGGC-3'; and GAP DH (glyceraldehyde-3-phosphate dehydrogenase, NM_02 3964) forward, 5 '-AGAAGGCTGGGGCTCATTTG-3' and reverse, 5'-AGGGGCCATCCACAGTCTTC-3'. Amplification was performed using the following cycling conditions: $50^{\circ} \mathrm{C}$ for two minutes (uracil-DNA glycosylase incubation), $95^{\circ} \mathrm{C}$ for 10 minutes, and 40 cycles of denaturation at $95^{\circ} \mathrm{C}$ for 15 seconds and annealing at $60^{\circ} \mathrm{C}$ for 30 seconds. All reactions were performed in triplicate. Gene expression was normalized to GAPDH (as an internal control). Data were analyzed by using the $2^{-\Delta \Delta \mathrm{Ct}}$ method.

\section{Double immunofluorescence staining for $\mathrm{TLR}_{4}$ and TICAM-2}

Double immunofluorescence staining was performed as described previously to explore the interaction between TLR $_{4}$ and TICAM-2 after IR [6,7]. Briefly, $10-\mu$ m-thick sections were incubated with a primary rabbit anti-TLR $\mathrm{T}_{4}$ antibody (1:800; Abcam, Cambridge, United States) and

Table 2 MicroRNAs (miRs) that were differentially expressed in the spinal cord of rats 72 hours after ischemia reperfusion (IR) injury compared with that in the sham-operated rats ( $n=$ three per group)

\begin{tabular}{|c|c|c|c|c|c|c|}
\hline \multirow[b]{2}{*}{$\mathrm{miR}$} & \multicolumn{2}{|c|}{ Average intensity of all probes (normalized) } & \multicolumn{2}{|c|}{ Standard deviation } & \multirow[b]{2}{*}{ Fold change } & \multirow[b]{2}{*}{$P$ value } \\
\hline & IR-72 hours & Sham-72 hours & IR-72 hours & Sham-72 hours & & \\
\hline \multicolumn{7}{|l|}{ Upregulated } \\
\hline rno-miR-144-3p & 0.1945 & 0.8025 & 0.3912 & 0.2997 & 4.1246 & 0.0139 \\
\hline rno-miR-466c-3p & 0.9230 & 1.5858 & 0.0982 & 0.2326 & 2.1179 & 0.0390 \\
\hline rno-miR-155-3p & 0.1711 & 0.3344 & 0.0599 & 0.2500 & 4.1709 & 0.0201 \\
\hline rno-miR-200b-3p & 0.0208 & 0.0627 & 0.1433 & 0.6803 & 3.1259 & 0.1591 \\
\hline \multicolumn{7}{|l|}{ Downregulated } \\
\hline rno-miR-10a-5p & 6.0399 & 3.4597 & 0.1390 & 0.0936 & 0.4957 & 0.0076 \\
\hline rno-miR-27a-3p ${ }^{a}$ & 1.1154 & 0.4575 & 0.0362 & 0.7307 & 0.3523 & 0.0276 \\
\hline rno-let-7a-5p & 0.7267 & 0.4315 & 1.5856 & 0.7777 & 0.4905 & 0.0206 \\
\hline rno-miR-382-3p & 2.8900 & 1.3146 & 0.1144 & 0.1230 & 0.4548 & 0.0017 \\
\hline rno-miR-21-5p & 3.7967 & 1.6388 & 0.1440 & 0.0352 & 0.4316 & 0.0024 \\
\hline rno-miR-411-3p & 1.8435 & 1.1789 & 0.0852 & 0.0306 & 0.4939 & 0.0020 \\
\hline rno-miR-547-5p & 0.3345 & 0.1405 & 0.2538 & 0.5311 & 0.4201 & 0.0410 \\
\hline rno-miR-702-3p & 0.3098 & 0.10918 & 0.3002 & 0.1005 & 0.4102 & 0.0206 \\
\hline rno-miR-10b-5p $p^{a}$ & 17.174 & 11.0843 & 0.0650 & 0.0903 & 0.4645 & 0.0021 \\
\hline rno-miR-23b-3pa & 10.736 & 6.6908 & 0.0318 & 0.0805 & 0.4623 & 0.0003 \\
\hline rno-miR-221-3p & 0.5148 & 0.2680 & 0.1642 & 0.2330 & 0.4520 & 0.0152 \\
\hline rno-miR-582-3p & 0.1262 & 0.0664 & 0.1170 & 0.2453 & 0.4526 & 0.0092 \\
\hline rno-miR-100-5p & 2.6130 & 1.3189 & 0.1617 & 0.2232 & 0.4504 & 0.0121 \\
\hline rno-miR-28-5p & 0.0722 & 0.0402 & 0.2261 & 0.0853 & 0.4557 & 0.0295 \\
\hline
\end{tabular}

Only the miRs with fold changes that were significantly different (upregulated $>2.0$ fold, downregulated $<0.5$ fold; $P<0.05$ ) are shown.

${ }^{a} \# \#$ : miRs that were differentially expressed in the spinal cord at both 24 and 72 hours after IR. 
a goat anti-TICAM-2 antibody (1:100; Santa Cruz Biotechnology, Santa Cruz, California, United States) overnight at $4^{\circ} \mathrm{C}$. After incubation with an Alexa 488conjugated donkey anti-rabbit immunoglobulin G (IgG) antibody (1:500; Molecular Probes, Eugene, United States) and an Alexa 594-conjugated donkey anti-goat IgG antibody (1:500; Molecular Probes), each for two hours at room temperature, images were captured using a Leica TCS SP2 laser scanning spectral confocal microscope (Leica Microsystems, Buffalo Grove, Illinois, United States).

\section{Western blot analysis}

The expression of $\mathrm{TLR}_{4}$, TICAM-2, and NF-kB p65 in spinal cord tissue was determined by western blot analyses. The rats' spinal cords were homogenized, and total proteins were purified using tissue and nuclear protein extraction reagents according to the manufacturer's instructions (KC-415 and KGP-150; KangChen, Shanghai, China). The antibodies against $\mathrm{TLR}_{4}$ (1:500; Abcam), TICAM-2 (1:500; Santa Cruz Biotechnology), and NF-кB p65 (1:500; Abcam) were used in this experiment, along with horseradish peroxidase-conjugated secondary antibodies (Bioss, Beijing, China). The scanned images were semi-quantitated using Quantity One software (Bio-Rad Laboratories, Milan, Italy).

\section{Measurement of Interleukin $-1 \beta$ content using ELISA}

The spinal cord was collected, homogenized, and then centrifuged. Interleukin (IL)-1 $\beta$ content was determined with an ELISA kit (R\&D Systems, Minneapolis, Minnesota,

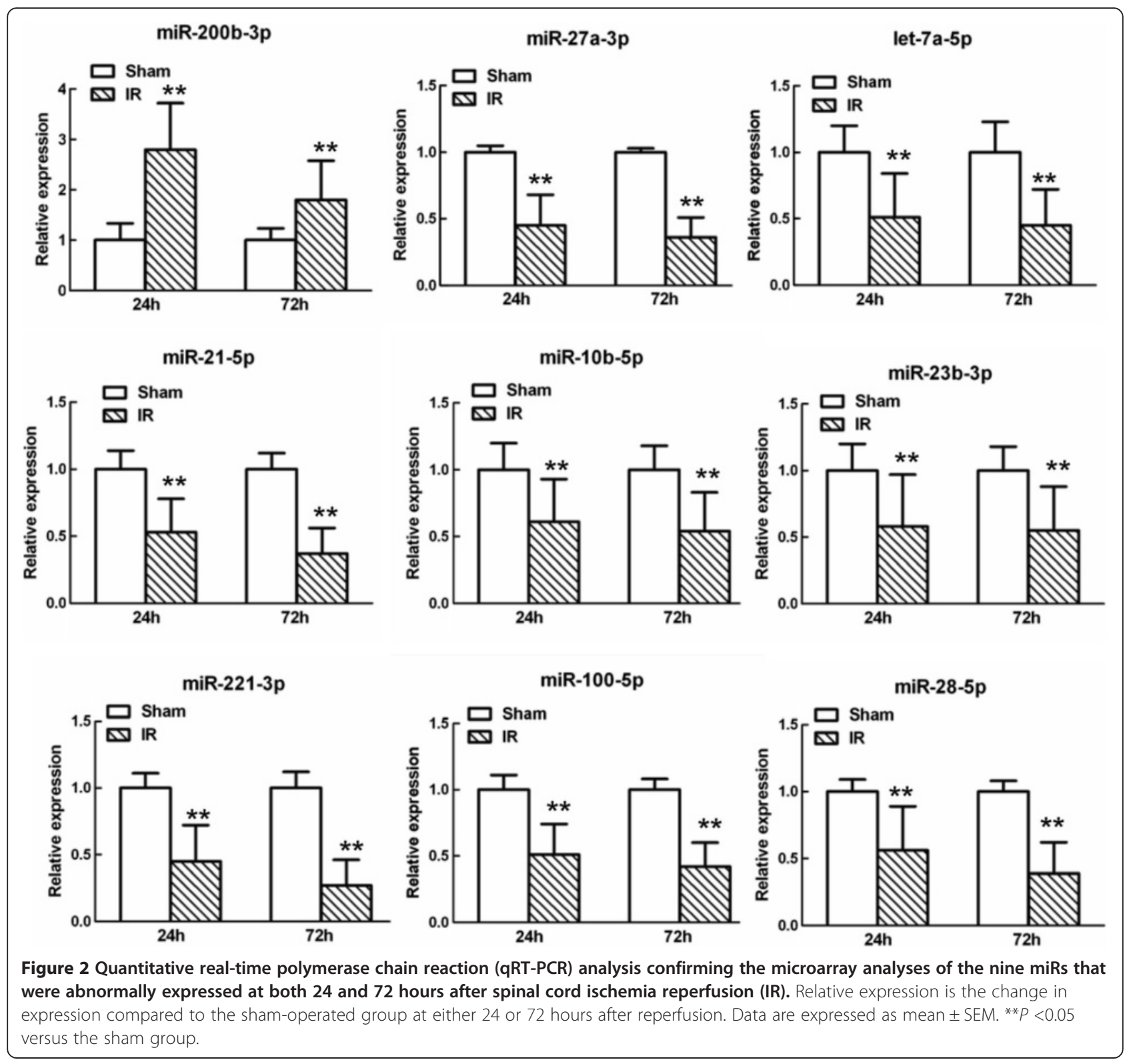


United States) according to the manufacturer's instructions. Absorbance (A) was measured at $450 \mathrm{~nm}$, and the IL-1 $\beta$ content of each sample was calculated based on a standard curve and was expressed in pictograms per milligram of total protein.

\section{Statistical analysis}

All data were expressed as mean \pm standard error of the mean (means \pm SEM) and were analyzed with SPSS software (version 17.0; SPSS Inc., Chicago, Illinois, United States). All variables measured in this study were normally distributed, and the groups were compared with Student's t-test or one-way analysis of variance (ANOVA), followed by Newman-Keuls post-hoc analysis. A $P$ value less than 0.05 was considered to be statistically significant.

\section{Results}

\section{Aberrant expression of miRs in the spinal cord after} ischemia reperfusion

To determine the potential involvement of miRs in the spinal cord after IR, we used microarray analysis to determine miR levels in the spinal cord at 24 and 72 hours after reperfusion. The results showed that compared with the sham group, in the IR group, three miRs were upregulated ( $>2.0$ fold) and $15 \mathrm{miRs}$ were downregulated $(<0.5$ fold) at 24 hours after surgery (Figure 1a, Table 1$)$, and four miRs were upregulated ( $>2.0$ fold) and $14 \mathrm{miRs}$ were downregulated $(<0.5$ fold) at 72 hours after surgery (Figure 1b, Table 2).

Nine of the miRs $(m i R-200 b-3 p, m i R-27 a-3 p$, let-7a5p, miR-21-5p, miR-10b-5p, miR-23b-3p, miR-221-3p, $m i R-100-5 p$, and $m i R-28-5 p$ ) were differently expressed at both 24 and 72 hours after reperfusion. Among them, $m i R-27 a-3 p$, let-7a-5p, miR-10b-5p, and $m i R-23 b-3 p$ have been shown to function as regulators of spinal cord development and remodeling, and have been implicated in diseases of the spinal cord $[20,21]$. Their abnormal expression was confirmed by quantitative real-time polymerase chain reaction (qRT-PCR) (Figure 2). The results showed that $m i R-27 a$ was expressed at significantly low levels after IR and continuously decreased with time $(P<0.05$, versus the sham group). In addition, searching the TargetScanHuman 6.2 and MicroCosm Targets version 5 databases revealed a perfect match to a miR-27a binding site in the 3'-UTR of the TICAM-2 gene (Figure 3a), which was confirmed by quantitative real-timeq RT-PCR (Figure $3 \mathrm{~b}$ ). Therefore, we hypothesized that downregulation of $m i R-27 a$ might play a role in mediating $\mathrm{TLR}_{4}$-mediated secondary inflammatory damage after IR by up-regulating the TICAM-2 transcript.

Intrathecal pretreatment with an miR-27a mimic and anti-miR oligonucleotides successfully regulated TICAM-2 expression after ischemia reperfusion

To explore the effects of a synthetic miR-27a mimic (mimic-27a), miR-27a AMO (AMO-27a), and a negative control mRNA (NC-27a) on TICAM-2 expression in the spinal cord after IR, we intrathecally injected mimic-27a, $A M O-27 a$, and $N C-27 a$ for three days before ischemia, and then examined both the mRNA and protein expression of TICAM-2 by RT-PCR and western blotting, respectively. As shown in Figure 4a, b, c, compared with the negative control $(N C-27 a)$, intrathecal injection with mimic-27a significantly prevented the IR-induced increases in TICAM-2 mRNA and protein expression at 24 and 72 hours after reperfusion, whereas injection with $A M O-27 a$ reversed these effects $(P<0.05$ versus the $N C-27 a$ group). Compared to the IR group,

a
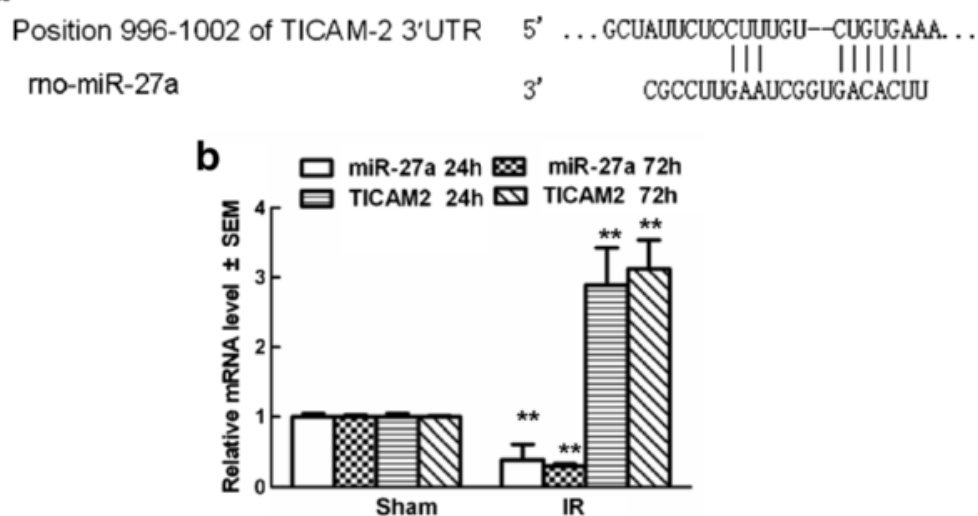

Figure 3 A putative target site of miR-27a located in the 3'-UTR of TICAM-2 mRNA was predicted by bioinformatics analysis. (a) The predicted miR-27a binding site in the 3'-untranslated region (UTR) of TICAM-2 in rats. (b) PCR analysis confirming TICAM-2 mRNA was abnormally expressed at both 24 and 72 hours after spinal cord ischemia reperfusion (IR). The profiles of TICAM-2 were expressed in an opposite manner as those of miR-27a. Relative expression is the change in expression compared to the sham-operated group at either 24 or 72 hours after reperfusion. Data are expressed as mean \pm SEM. ${ }^{* *} P<0.05$ versus the sham group. Results shown are representative data from three separate experiments. 


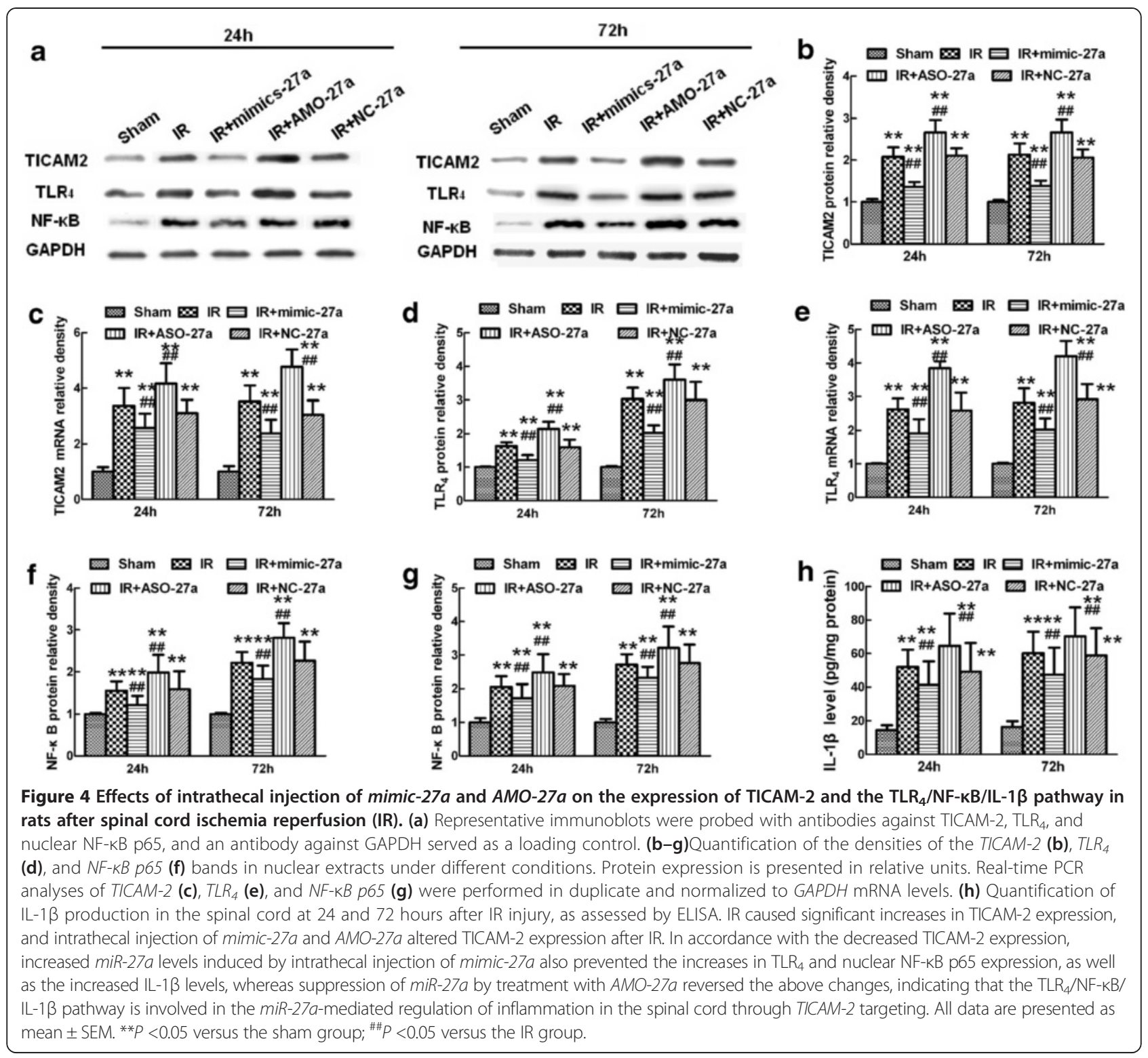

intrathecal injection of $\mathrm{NC}-27 \mathrm{a}$ before ischemia induced no obvious differences at 24 and 72 hours after reperfusion $(P>0.05$ versus the IR group).

\section{Expression and colocalization of TLR $\mathrm{T}_{4}$ and TICAM-2 after ischemia reperfusion}

TICAM-2 is a cytoplasmic protein that is known to be associated with $\mathrm{TLR}_{4}$ signaling. The relationship between TICAM-2 and TLR $_{4}$ expression in the spinal cord 24 and 72 hours after reperfusion was examined by double immunofluorescence staining. As shown in Figure 5a, the level of membrane-bound $\mathrm{TLR}_{4}$ immunostaining was similar to that of cytoplasmic TICAM-2 immunostaining in the neurons and glial cells of injured spinal cords, but not in sham-operated ones, which confirmed that $\mathrm{TLR}_{4}$ was indeed upregulated when TICAM-2 was upregulated during IR. Quantification of TICAM-2 expression is shown in Figure $5 \mathrm{~b}(P<0.05$ versus the IR group).

Conversely, compared to the IR group, increased miR-27a levels by pretreatment with mimic-27a reduced TICAM-2 immunoreactivity and the number of double-labeled cells in lumbar spinal cords during the 72-hour follow-up period. In contrast, much stronger TICAM-2 immunoreactivity and more double-labeled cells were detected in the spinal cords of rats pretreated with $A M O-27 a(P<0.05$ versus the IR group). No significant difference was observed in operated rats with or without $N C-27 a$ treatment at either 24 or 72 hours $(P>0.05$ versus the IR group). Quantification of the double-labeled cells is shown in Figure 5c. 


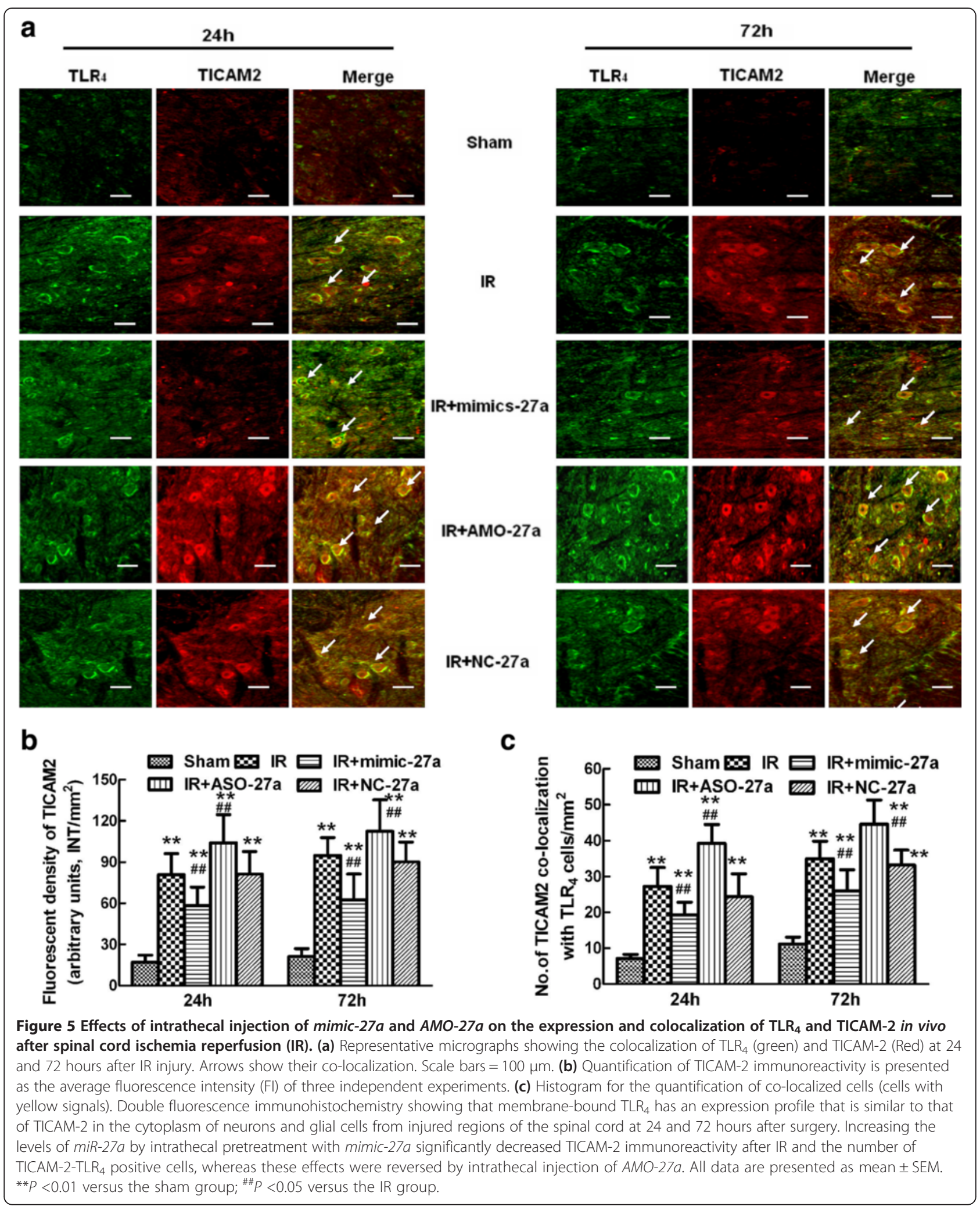


Intrathecal pretreatment with mimic-27a attenuated blood-spinal cord barrier (BSCB) leakage after ischemia reperfusion

EB content and fluorescent dye were used to assess IR-induced BSCB disruption as described previously $[6,7,22]$. BSCB permeability was visualized by EB extravasation. As shown in Figure 6a, compared with the sham group, IR caused a marked increase in EB extravasation at 24 and 72 hours after reperfusion $(P<0.05$ versus the sham group). Intrathecal infusion of mimic-27a attenuated BSCB dysfunction, as evidenced by the decreased EB extravasation and fluorescent dye content at 24 and 72 hours after reperfusion, whereas intrathecal infusion of $A M O-27 a$ synergistically exacerbated BSCB leakage $(P<0.05$ versus the IR group). There were no detectable differences in EB extravasation between the IR group and the group pretreated with $N C-27 a$ at all time points $(P>0.05)$. The quantification of $\mathrm{EB}$ content in the spinal cord shown in Figure $6 \mathrm{~b}$ and the fluorescent densities shown in Figure 6c confirmed the above results.
Intrathecal pretreatment with mimic-27a inhibited TLR ${ }_{4} /$ NF-KB/IL-1 $\beta$ activation after ischemia reperfusion

To further confirm that $m i R-27 a$ is involved in $\mathrm{TLR}_{4}$ activation by regulating the expression of TICAM- 2 after IR, we examined the mRNA and protein levels of $\mathrm{TLR}_{4}$, $\mathrm{NF}-\mathrm{kB}$, and the downstream inflammatory cytokine IL-1 $\beta$ by ELISA. In agreement with the double immunofluorescence TICAM-2 and $\mathrm{TLR}_{4}$ staining, intrathecal pretreatment with mimic-27a reduced the IR-induced $\mathrm{TLR}_{4}$ and NF-kB mRNA and protein levels at 24 and 72 hours after reperfusion (Figure 4a, d, e, f; $P<0.05$ versus the IR group). In contrast, intrathecal infusion of AMO-27a increased $\mathrm{TLR}_{4}$ and NF-kB mRNA and protein levels $(P<0.05$ versus the IR group) at all time points. Compared to the IR group, there were no detectable differences in $\mathrm{TLR}_{4}$ and NF- $\mathrm{kB}$ expression when animals were pretreated with $N C-27 a(P>0.05)$. Furthermore, levels of the downstream inflammatory cytokine IL-1 $\beta$ also increased with $\mathrm{TLR}_{4}$ and NF-kB increasing (Figure 4h; $P<0.05$ versus the IR group). These results are consistent with the hypothesis that the IR-mediated reduction

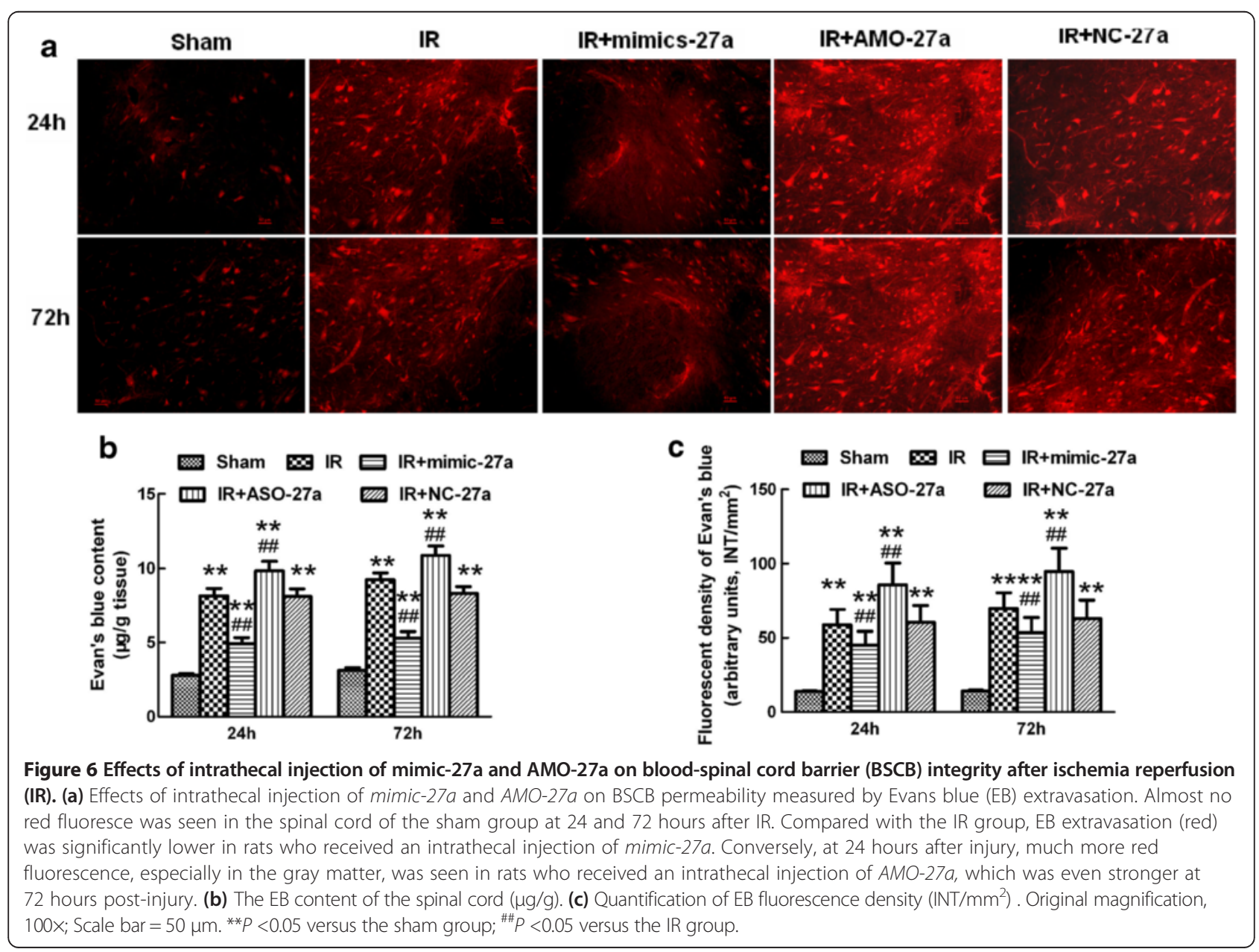


in $m i R-27 a$ down-regulates TICAM-2 expression, which in turn influences $\mathrm{TLR}_{4}$ signaling and the activation of downstream inflammatory cytokines.

\section{Discussion}

The BSCB is a physical and biochemical barrier between the circulation and the spinal cord that plays an important role in the regulation of spinal cord homeostasis. Disruption of BSCB integrity after IR injury has been reported to trigger alterations in the spinal microenvironment, allowing penetration of both inflammatory cytokines and immune cells into the spinal cord, which determines the prognosis of patients with IR injury $[6,7,22]$. Accumulating evidence indicates that some of the major effectors in this inflammatory damage to BSCB are engagement with tolllike receptors (the TLRs) signal pathway [5-8,23]. Recently, many studies have shown that individual miRs, endogenous, non-coding, single-stranded, small RNAs that are widespread in eukaryotic organisms, are capable of affecting numerous target mRNAs and effectively regulating their gene expression $[8,15,18,19]$. Altering the expression of miRs greatly affects the pathogenesis of IR and its functional outcome $[16,24]$. Therefore, in this present study, we provide evidences that miRs attenuate BSCB permeability, and our findings expand the understanding of the molecular mechanisms underlying $\mathrm{TLR}_{4}$-mediated inflammatory responses. To the best of our knowledge, this is the first study to document a miR-27a target related to the $\mathrm{TLR}_{4}$ pathway in spinal cord IR injury.

MiRs are a class of sophisticated gene expression regulators with the unique ability to prevent the translation of and/or degrade their corresponding target mRNAs by inhibiting translation and promoting cleavage, respectively. MiRs act as key regulators in a wide variety of biological processes, including cell proliferation, differentiation, apoptosis, and organ development, and have also been implicated in inflammatory diseases $[25,26]$. Previous studies showed that some miRs may regulate genes associated with TLR signaling pathways; the mRNA expression of five adaptor and interacting proteins (CD14, HSPA1a, Pglyrp-1, MD-1, and TICAM-2) were significantly upregulated following traumatic brain injury (TBI) $[8,23]$. In this study, using a miRs microarray screening approach, we found that $m i R-27 a$ expression was significantly altered at both $24 \mathrm{~h}$ and 72 hours after IR, and such results were as confirmed by qRT-qPCR (Figure 2).

In animals, recognition of miR response elements only requires a continuous 6six-base pair "seed match"' near the 3'-UTR of its target mRNA [27]. Given this, many target genes of miR-27a have been identified, some of which were showed to participated in innate immune response and inflammatory responses [28-31]. Whether miR-27a specifically target TICAM-2 in vivo remainsed to be tested. It hasis recently been shown that pretreatment with miR mimics and AMOs was is one of the most common and useful methods to regulate the expression levels of miRs $[16,17,28,29]$. He et al. reported that injecting miR mimics 48 hours before IR greatly downregulated the levels of apoptosis-related genes, whereas injecting the corresponding AMOs upregulated their expression [17]. Our in vivo data, shown in Figure 4a, b, c, are in close accordance with previous studies, showing that compensating for decreased $m i R$ $27 a$ levels by continuous intrathecal injection of mimic$27 a$ into the subarachnoid space of IR model rats, reduced the mRNA and protein expression of TICAM-2 by inhibiting the translation and/or promoting the degradation of its mRNA. Continuous intrathecal injection of $A M O-27 a$ before ischemia clearly abrogated reversed such changes. However, no obvious changes were detected when model animals were pretreated with $N C-27 a$, suggesting that miR-27a directly modulates TICAM-2 expression in vivo, and that modulation of $m i R-27 a$ is particularly important in the pathophysiology of IR.

TICAM-2 is a cytoplasmic protein that structurally resembles the MAL/TIRAP adaptor that links $\mathrm{TLR}_{4}$ and $\mathrm{MyD}_{88}$ and functionally transmits $\mathrm{TLR}_{4}$ signaling to TICAM-1 $[9,32]$. Given the bridging action between TLR 4 and TRIF, TICAM-2 coordinates the inflammatory response to a pathogen challenge [33]. Thus, it is easy to postulate that IR-induced aberrant TICAM-2 expression might be closely associated with $\mathrm{TLR}_{4}$ activation, subsequent NF- $\mathrm{kB}$ relocation to the nucleus, and the release of downstream proinflammatory cytokines $[9,32,34]$. Consistent with this, here, we observe that $\mathrm{TLR}_{4}$ expression is also significantly upregulated when TICAM-2 expression increased and miR-27a decreased at both 24 and 72 hours after IR (Figure 4a, b, c, d, e). In addition, the double immunofluorescence staining in Figure 5 shows that the signal for $\mathrm{TLR}_{4}$, a membrane-bound receptor, is greatly upregulated coincident along with increased cytoplasmic staining of TICAM-2 in neuronal and glial cells of ischemic spinal cords. Therefore, treatments that decrease TICAM-2 and inhibit the inflammatory stimulation of $\mathrm{TLR}_{4}$ might be a novel intervention for IR $[9,32,33,35]$. We then identifiedy the mechanism underlying miR-dependent regulation of TICAM-2 expression. Intrathecal injection of mimic-27a prevented the increase in TICAM-2 immunoreactivity and the number of double-labeled cells, whereas intrathecal injection of $A M O-27 a$ reversed these effects. In accordance with these observed effects of mimic-27a and AMO-27a on TICAM-2, we observed similar expression profiles for $\mathrm{TLR}_{4}$, NF-kB, and the proinflammatory cytokine IL-1 $\beta$ at 24 and 72 hours post-injury, providing direct evidence that IR-induced $\mathrm{TLR}_{4}$ activation is significantly influenced by the expression of TICAM-2. 
Proinflammatory cytokines are important molecules in the immune system that have been implicated in alterations of $\mathrm{BSCB}$ integrity $[6,7,36,37]$. Breakdown of the BSCB allows exogenous pathogens and circulating immune cells to enter the spinal cord, which has numerous consequences, including neuronal loss, central sensitization, and glial remodeling [22,36]. Inhibiting inflammatory damage to the BSCB has been postulated as thea key to protection in IR. Our present study provides clear evidence for the protective effects of specifically increasing $m i R-27 a$ expression. An Iinjection of mimic-27a attenuated BSCB dysfunction, which was manifested as reduced fluorescent dye and EB extravasation, and was suppressed by the injection of $A M O-27 a$; this finding indicatesd that $m i R-27 a$ regulates inflammatory damage to the BSCB by targeting the TICAM- 2 mRNA and the $\mathrm{TLR}_{4} / \mathrm{NF}-\mathrm{kB} / \mathrm{IL}-1 \beta$ pathway.

Significantly, one major character of miRs is that a single miR is capable of regulating the expression of many target genes, whereas a target gene can also be regulated by several miRs $[11,13,26]$. Thus, it is very possible to gain different or even contradictory expressions when exploring the same miR in differently experimental conditions $[20,28,38,39]$. For example, in the recsent study of Young et al. showed the opposite role of miR-27a in the regulation of vascular leaking by targeting vascular endothelium (VE)-cadherin in the endothelium [38]. On the other hand, some studies showed an upregulation of miR-27a for systemic inflammation, instead of the down-regulation as described in this study in the event of systemic inflammation $[28,39]$. These disagreements might be caused by the different observation time points, which are consistent with the descriptions of that no changes being observed in miR-27a expression for at 6 hours, instead of downregulation for at 24 hours after lipopolysaccharide (LPS) treatments [28]. Moreover, the net effects of miRs observed in in vivo experiments were also influenced by the complicated internal environment $[20,40]$. Luxenhofer $\mathrm{G}$ et al. emphasized the importance of miRs in regulating the molecular network specifying the generation of neuronal diversity in the developing chick spinal cord [40]. Ziu M et al. also showed significantly different expression levels of miRs in prolonged compression injury compared to those in short compression injury [20]. Furthermore, different expressions of miRs were reported in different regions of the injured spinal cord, even under the same experimental conditions [20]. Thus, aseptically inflammatory responses during spinal cord IR injury could not be exactly equivalent to directly proinflammatory stimuli with LPS in vitro as well as the results obtained from the lung tissue. Further in vitro and in vivo studies still need to be conducted to identify the correlation between $m i R-27 a$ and the corresponding target genes in the mode of inflammatory or anti-inflammatory actions to better elucidate the mechanism and provide potential therapeutic targets for IR.

Taken together, we identify TICAM-2 as a novel target of $m i R-27 a$ and show that downregulation of $m i R$ $27 a$ promotes IR-induced inflammatory damage to the $\mathrm{BSCB}$ by facilitating activation of the $\mathrm{TLR}_{4} / \mathrm{NF}-\mathrm{kB} / \mathrm{IL}-1 \beta$ signaling pathway.

\begin{abstract}
Abbreviations
AMO: Anti-miRNA oligonucleotides; BSA: Bovine serum albumin; BSCB: Blood-spinal cord barrier; EB: Evans blue; GAPDH: Glyceraldehyde-3-phosphate dehydrogenase; IR: Ischemia-reperfusion; ID: Inside diameter;

IgG: Immunoglobulin G; IL: Interleukin; INT: Intensity; IRF3: Interferon regulatory factor 3; LPS: Lipopolysaccharide; miRs: microRNAs; $M_{1 y} D_{88}$ : Myeloid differentiation factor $88 ;$ Mal/TIRAP: MyD88 adaptor-like; NC: Negative control; NF-kB: Nuclear factor kappa-B; OD: Outside diameter; qRT-PCR: Quantitative real-time polymerase chain reaction; SEM: Standard error of the mean; TBI: Traumatic brain injury; TLR: Toll-like receptor 4; TICAM-2: Toll-like receptor adaptor molecule 2; TRIF: TIR domain-containing adaptor-inducing IFN- $\beta$; UTR: Untranslated region.
\end{abstract}

\section{Competing interests}

The authors declare that they have no competing interests.

\section{Authors' contributions}

$\mathrm{X}-\mathrm{QL}, \mathrm{Z}-\mathrm{LW}$, and BF participated in animal care and made the animal models. $X-Q L, Z-L W$, and W-FT prepared and sectioned tissues and performed most of the immunohistochemistry assays. X-QL, BF, and W-FT performed the western blot assays and the statistical analysis. Z-LW, BF, and H-WL conducted the miRNA microarray analysis and luciferase assays. HM guided the model design and study design. H-WL gave important directions for data analysis and manuscript writing. All authors read and approved the final manuscript.

\section{Acknowledgments}

Funding for this project was provided by the Doctoral Research Fund of Liaoning Province (grant number: 20141035) and the Natural Science

Foundation of China (grant number: 81271370).

Received: 21 July 2014 Accepted: 13 January 2015

Published online: 07 February 2015

\section{References}

1. Lim KH, Connolly M, Rose D, Siegman F, Jacobowitz I, Acinapura A, et al. Prevention of reperfusion injury of the ischemic spinal cord: use of recombinant superoxide dismutase. Ann Thorac Surg. 1986;42(3):282-6.

2. Fan L, Wang K, Shi Z, Die J, Wang C, Dang X. Tetramethylpyrazine protects spinal cord and reduces inflammation in a rat model of spinal cord ischemia-reperfusion injury. J Vasc Surg. 2011;54(1):192-200.

3. Zhu P, Li JX, Fujino M, Zhuang J, Li XK. Development and treatments of inflammatory cells and cytokines in spinal cord ischemia-reperfusion injury. Mediators Inflamm. 2013;2013:701970.

4. Ni B, Cao Z, Liu Y. Glycyrrhizin protects spinal cord and reduces inflammation in spinal cord ischemia-reperfusion injury. Int J Neurosci. 2013;123(11):745-51.

5. Bell MT, Puskas F, Agoston VA, Cleveland Jr JC, Freeman KA, Gamboni F, et al. Toll-like receptor 4-dependent microglial activation mediates spinal cord ischemia-reperfusion injury. Circulation. 2013;128(11 Suppl 1):S152-6.

6. Li XQ, Lv HW, Tan WF, Fang B, Wang H, Ma H. Role of the TLR4 pathway in blood-spinal cord barrier dysfunction during the bimodal stage after ischemia/reperfusion injury in rats. J Neuroinflammation. 2014;11:62.

7. Li XQ, Wang J, Fang B, Tan WF, Ma H. Intrathecal antagonism of microglial TLR4 reduces inflammatory damage to blood-spinal cord barrier following ischemia/reperfusion injury in rats. Mol Brain. 2014;7(1):28.

8. Hua F, Wang J, Ishrat T, Wei W, Atif F, Sayeed I, et al. Genomic profile of Toll-like receptor pathways in traumatically brain-injured mice: effect of exogenous progesterone. J Neuroinflammation. 2011;8:42.

9. Oshiumi H, Sasai M, Shida K, Fujita T, Matsumoto M, Seya T. TIR-containing adapter molecule (TICAM)-2, a bridging adapter recruiting to Toll-like receptor 4 TICAM-1 that induces interferon- $\beta$. J Biol Chem. 2003;278:49751-62. 
10. Yang M, Yuan S, Huang S, Li J, Xu L, Huang H, et al. Characterization of bbtTICAM from amphioxus suggests the emergence of MyD88-independent pathway in basal chordates. Cell Res. 2011;21(10):1410-23.

11. Redell JB, Liu Y, Dash PK. Traumatic brain injury alters expression of hippocampal microRNAs: potential regulators of multiple pathophysiological processes. J Neurosci Res. 2009;87(6):1435-48.

12. Jin L, Wu Z, Xu W, Hu X, Zhang J, Xue Z, et al. Identifying gene expression profile of spinal cord injury in rat by bioinformatics strategy. Mol Biol Rep. 2014;41(5):3169-77.

13. Brandenburger T, Castoldi M, Brendel M, Grievink H, Schlösser L, Werdehausen $\mathrm{R}$, et al. Expression of spinal cord microRNAs in a rat model of chronic neuropathic pain. Neurosci Lett. 2012;506(2):281-6.

14. Bhalala OG, Srikanth M, Kessler JA. The emerging roles of microRNAs in CNS injuries. Nat Rev Neurol. 2013;9(6):328-39.

15. Guo D, Liu J, Wang W, Hao F, Sun X, Wu X, et al. Alteration in abundance and compartmentalization of inflammation-related miRNAs in plasma after intracerebral hemorrhage. Stroke. 2013;44(6):1739-42.

16. Hinkel R, Penzkofer D, Zühlke S, Fischer A, Husada W, Xu QF, et al. Inhibition of microRNA-92a protects against ischemia/reperfusion injury in a large-animal model. Circulation. 2013;128(10):1066-75.

17. He B, Xiao J, Ren AJ, Zhang YF, Zhang H, Chen M, et al. Role of miR-1 and miR-133a in myocardial ischemic postconditioning. J Biomed Sci. 2011;18:22.

18. Yunta M, Nieto-Díaz M, Esteban FJ, Caballero-López M, Navarro-Ruíz R, Reigada $\mathrm{D}$, et al. MicroRNA dysregulation in the spinal cord following traumatic injury. PLoS One. 2012;7(4):e34534

19. Jee MK, Jung JS, Choi Jl, Jang JA, Kang KS, Im YB, et al. MicroRNA 486 is a potentially novel target for the treatment of spinal cord injury. Brain. 2012;135(Pt 4):1237-52

20. Ziu M, Fletcher L, Savage JG, Jimenez DF, Digicaylioglu M, Bartanusz V. Spatial and temporal expression levels of specific microRNAs in a spinal cord injury mouse model and their relationship to the duration of compression. Spine J. 2014;14:353-60.

21. Zuo Q, Xu JJ. Specific expression of microrna in different tissues of nervous system and expression changes in nerve regeneration. Sheng Li Ke Xue Jin Zhan. 2011:42:261-8

22. Fang B, Li XM, Sun XJ, Bao NR, Ren XY, Lv HW, et al. Ischemic preconditioning protects against spinal cord ischemia-reperfusion injury in rabbits by attenuating blood spinal cord barrier disruption. Int J Mol Sci. 2013;14:10343-54

23. Nahid MA, Satoh M, Chan EK. MicroRNA in TLR signaling and endotoxin tolerance. Cell Mol Immunol. 2011;8(5):388-403.

24. $\mathrm{Hu} J \mathrm{~J}, \mathrm{LV}$ GH, Yin BL. Altered MicroRNA expression in the ischemic-reperfusion spinal cord with atorvastatin therapy. J Pharmacol Sci. 2013;121:343-6.

25. O'Connell RM, Rao DS, Baltimore D. microRNA regulation of inflammatory responses. Annu Rev Immunol. 2012;30:295-312.

26. Ksiazek-Winiarek DJ, Kacperska MJ, Glabinski A. MicroRNAs as novel regulators of neuroinflammation. Mediators Inflamm. 2013;2013:172351.

27. Selbach M, Schwanhäusser B, Thierfelder N, Fang Z, Khanin R, Rajewsky N. Widespread changes in protein synthesis induced by microRNAs. Nature. 2008;455(7209):58-63.

28. Xie N, Cui H, Banerjee S, Tan Z, Salomao R, Fu M, et al. miR-27a regulates inflammatory response of macrophages by targeting IL-10. J Immunol. 2014;193(1):327-34.

29. Wang T, Li M, Guan J, Li P, Wang H, Guo Y, et al. MicroRNAs miR-27a and miR-143 regulate porcine adipocyte lipid metabolism. Int J Mol Sci. 2011;12:7950-9.

30. Liu G, Cao P, Chen H, Yuan W, Wang J, Tang X. MiR-27a regulates apoptosis in nucleus pulposus cells by targeting PI3K. PLoS One. 2013;8(9):e75251.

31. Liu G, Abraham E. MicroRNAs in immune response and macrophage polarization. Arterioscler Thromb Vasc Biol. 2013;33(2):170-7.

32. Enokizono Y, Kumeta H, Funami K, Horiuchi M, Sarmiento J, Yamashita K, et al. Structures and interface mapping of the TIR domain-containing adaptor molecule involved in interferon signaling. Proc Natl Acad Sci U S A. 2013;110:19908-13.

33. Fitzgerald KA, Rowe DC, Barnes BJ, Caffrey DR, Visintin A, Latz E, et al. LPS-TLR4 signaling to IRF-3/7 and NF-kappaB involves the toll adapters TRAM and TRIF. J Exp Med. 2003;198:1043-55.

34. Verstak B, Stack J, Ve T, Mangan M, Hjerrild K, et al. The TLR signaling adaptor TRAM interacts with TRAF6 to mediate activation of the inflammatory response byTLR4. J Leukoc Biol. 2014;96:427-36.
35. Zughaier SM, Zimmer SM, Datta A, Carlson RW, Stephens DS. Differential induction of the Toll-like receptor 4-MyD88-dependent and -independent signaling pathways by endotoxins. Infec Immun. 2005;73:2940-50.

36. Echeverry S, Shi XQ, Rivest $S$, Zhang J. Peripheral nerve injury alters blood-spinal cord barrier functional and molecular integrity through a selective inflammatory pathway. J Neurosc. 2011;31(30):10819-28.

37. Beggs S, Liu XJ, Kwan C, Salter MW. Peripheral nerve injury and TRPV1-expressing primary afferent C-fibers cause opening of the blood-brain barrier. Mol Pain. 2010;6:74

38. Young JA, Ting KK, Li J, Moller T, Dunn L, Lu Y, et al. Regulation of vascular leak and recovery from ischemic injury by general and VE-cadherinrestricted miRNA antagonists of miR-27. Blood. 2013;122(16):2911-9.

39. Wang Z, Ruan Z, Mao Y, Dong W, Zhang Y, Yin N, et al. miR-27a is up regulated and promotes inflammatory response in sepsis. Cell Immunol. 2014;290(2):190-5.

40. Luxenhofer G, Helmbrecht MS, Langhoff J, Giusti SA, Refojo D, Huber AB. MicroRNA-9 promotes the switch from early-born to late-born motor neuron populations by regulating Onecut transcription factor expression. Dev Biol. 2014;386(2):358-70.

\section{Submit your next manuscript to BioMed Central and take full advantage of:}

- Convenient online submission

- Thorough peer review

- No space constraints or color figure charges

- Immediate publication on acceptance

- Inclusion in PubMed, CAS, Scopus and Google Scholar

- Research which is freely available for redistribution 